\title{
Erratum to: Low expression of ULK1 is associated with operable breast cancer progression and is an adverse prognostic marker of survival for patients
}

\author{
Jun Tang ${ }^{1,2} \cdot$ Rong Deng $^{1} \cdot$ Rong-Zhen Luo ${ }^{1,3} \cdot$ Guo-Ping Shen $^{1} \cdot$ Mu-Yan Cai ${ }^{1,3}$. \\ Zi-Ming Du ${ }^{1,3} \cdot$ Shan Jiang ${ }^{1}$ Ming-Tian Yang ${ }^{1,2} \cdot$ Jian-Hua Fu ${ }^{1,4}$. \\ Xiao-Feng Zhu' ${ }^{1}$
}

Published online: 9 March 2016

(C) Springer Science+Business Media New York 2016

\section{Erratum to: Breast Cancer Res Treat (2012) \\ 134:549-560 \\ DOI 10.1007/s10549-012-2080-y}

In the original publication of the article, Fig. $5 \mathrm{c}$ was published incorrectly. The authors apologize for this error and the correct Fig. 5c is given below.

The online version of the original article can be found under doi:10.1007/s10549-012-2080-y.

Jian-Hua Fu

fujh@sysucc.org.cn

$\bowtie$ Xiao-Feng Zhu

zhuxfeng@mail.sysu.edu.cn

1 State Key Laboratory of Oncology in South China, Cancer Center, Sun Yat-sen University, Guangzhou, China

2 Department of Breast Oncology, Cancer Center, Sun Yat-sen University, Guangzhou, China

3 Department of Pathology, Cancer Center, Sun Yat-sen University, Guangzhou, China

4 Department of Thoracic Surgery, Cancer Center, Sun Yat-sen University, Guangzhou, China 

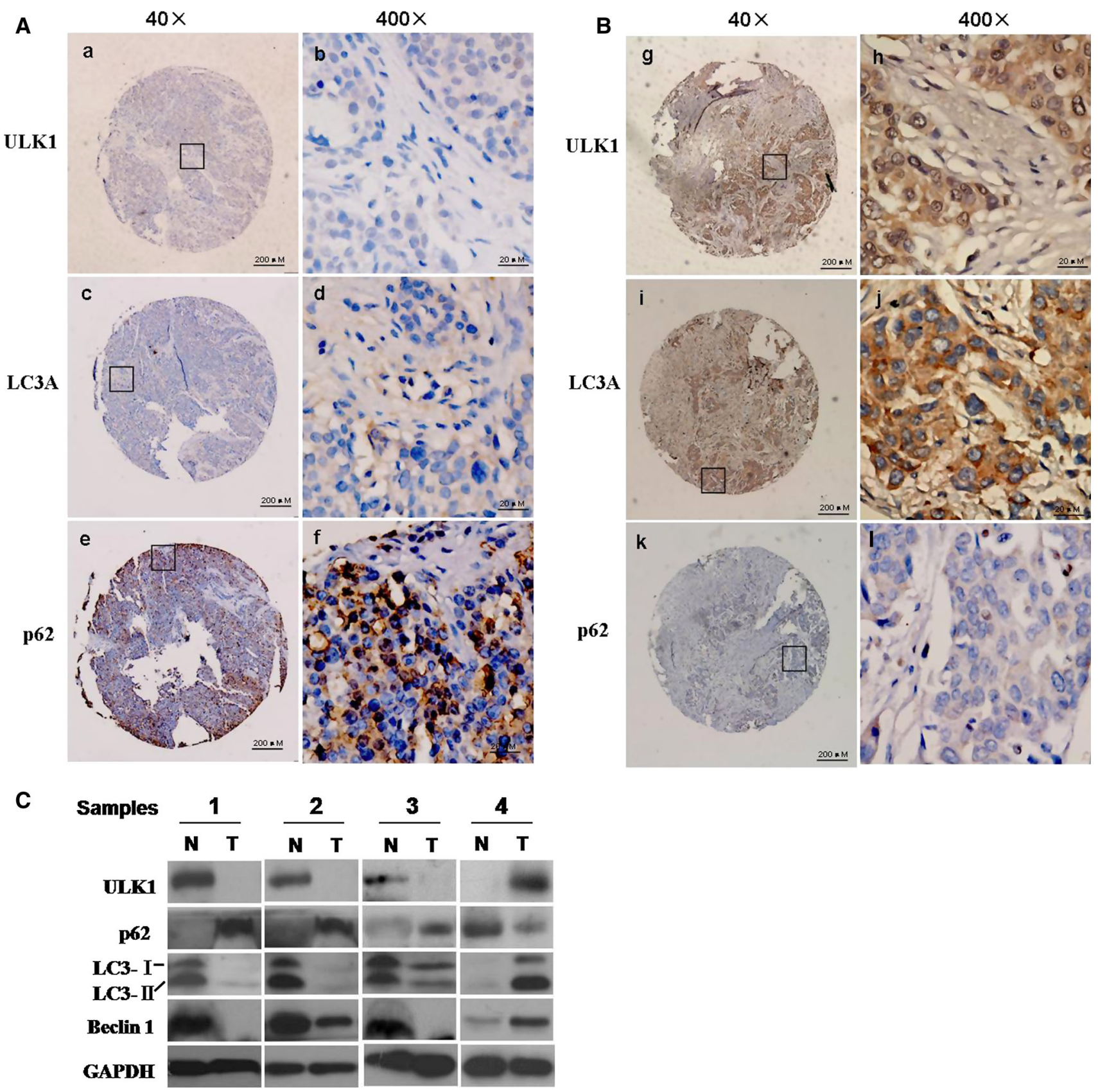

Fig. 5 Correlation between the expression of ULK1 and autophagic activity in breast cancer tissues. A Low expression of $\operatorname{ULK} 1(a, b)$ associated with low expression of LC3A $(c, d)$ and high expression of p62 $(e, f)$ was observed on the serial section of the same case 188. B High expression of ULK1 $(g, h)$ associated with high expression of

LC3A $(i, j)$ and low expression of p62 $(k, l)$ was observed on the serial section of the same case 281. C Western blot results of ULK1, p62, LC3 and Beclin1 protein expression in four pairs of matched breast tissue. GADPH was used as loading control 